\title{
RANDOM PRODUCTS OF MAPS SYNCHRONIZING ON AVERAGE
}

\author{
EDGAR MATIAS AND ÍTALO MELO
}

\begin{abstract}
We present a necessary and sufficient condition for a random product of maps on a compact metric space to be (strongly) synchronizing on average.
\end{abstract}

\section{INTRODUCTION}

Let $(\Omega, \mathscr{F}, \mathbb{P}, \theta)$ be a measure-preserving dynamical system and $(X, d)$ a compact metric space (endowed with the Borel $\sigma$-algebra $\mathscr{B}$ ). Let $f: \Omega \times X \rightarrow X$ be a $\mathscr{F} \otimes \mathscr{B} / \mathscr{B}$-measurable map. A random product of maps on $X$ over the measurepreserving dynamical system $(\Omega, \mathscr{F}, \mathbb{P}, \theta)$ is a map $\varphi: \mathbb{N} \times \Omega \times X \rightarrow X$ defined by

$$
\varphi(n, \omega, x) \stackrel{\text { def }}{=} f_{\theta^{n-1}(\omega)} \circ \cdots \circ f_{\omega}(x) \stackrel{\text { def }}{=} f_{\omega}^{n}(x) \text { for } n \geq 1,
$$

where $f_{\omega}: X \rightarrow X$ is a family of maps given by $f_{\omega}(x) \stackrel{\text { def }}{=} f(\omega, x)$. We often refer to $\varphi$ as a random product of maps $f_{\theta^{i}(\omega)}$ on $X$ over the measure-preserving dynamical system $(\Omega, \mathscr{F}, \mathbb{P}, \theta)$.

In this paper we present a necessary and sufficient condition to obtain synchronization of the random orbits $f_{\omega}^{n}(x)$, that is, random orbits of different initial points converging to each other. The synchronization effect was first observed by Huygens [13] in the movement of two pendulum clocks hanging from a wall and since then has been investigated in several areas, see 20. Despite the vast literature on this subject, most of the results are experimental. The first rigorous results about synchronization of independent and identically distributed (i.i.d.) random maps goes back to [4, 10, and more recently the synchronization on the circle has been intensively studied, see [11, 12, 16, 18].

For random products there are several ways to introduce the notion of synchronization. In this paper we consider convergence of orbits on average. First we recall that a random product $\varphi$ is synchronizing on average according to 12 if for every $x, y \in X$, for $\mathbb{P}$-almost every $\omega$ it holds

$$
\lim _{n \rightarrow \infty} \frac{1}{n} \sum_{i=0}^{n-1} d\left(f_{\omega}^{i}(x), f_{\omega}^{i}(y)\right)=0 .
$$

2000 Mathematics Subject Classification. 37C70, 37H05, 37C40.

Key words and phrases. Random product of maps, synchronization, invariant graphs, skew products, invariant measures.

E.M is currently supported by PNPD CAPES. This work was partially completed while E.M was supported by PCI award 312487/2016-9 at IMPA and I.M was supported by a CNPq-Brazil doctoral fellowship at IMPA. The authors warmly thank K. Gelfert and Lorenzo Díaz for their useful comments on this paper. 
We introduce the following slightly stronger concept, the difference being a "change of quantifiers". We say that $\varphi$ is strongly synchronizing on average if for $\mathbb{P}$-almost every $\omega$ it holds (1.1) for every $x, y \in X$.

The literature suggests that synchronization is often related with the existence of an invariant graph. An invariant map is a measurable map $\Phi: \Omega \rightarrow X$ such that

$$
f_{\omega}(\Phi(\omega))=\Phi(\theta(\omega))
$$

for $\mathbb{P}$-almost every $\omega$. The graph of an invariant map is called an invariant graph. We observe that invariant maps sometimes provide relevant information about the random product. In some cases an invariant map is given explicitly by

$$
\Phi(\omega) \stackrel{\text { def }}{=} \lim _{n \rightarrow \infty} f_{\theta^{-1}(\omega)} \circ \cdots \circ f_{\theta^{-n}(\omega)}(p)
$$

where the limit exists for $\mathbb{P}$-almost every $\omega$ in $\Omega$ and it is independent of the point $p \in X$. For instance, in the theory of contracting iterated functions system the map $\Phi$ is well-defined for every $\omega \in \Omega$ and (see [14, Section 3.1]) it is the unique invariant map. Note that in this case the image of $\Phi$ is the Hutchinson attractor and its distribution is the unique stationary measure (supported on this attractor) 1 . This indicates the importance of invariant maps for the description of dynamical properties of the system. To mention some more general results in the i.i.d. case for systems which are non-contracting, perhaps one of the first in this spirit is due to Letac [17. The key hypothesis in [17] is that the map in (1.3) is well-defined for $\mathbb{P}$-almost every $\omega$ in $\Omega$ and it is proved that the distribution of $\Phi$ is the unique stationary measure. We point out that the property $\Phi$ is well-defined (almost everywhere) is, in fact, a consequence of much weaker topological conditions such as the so-called splitting property in [9] or the very classical condition of being contracting on average in [6]. For further results on the existence of invariant maps see the comments which follow Theorem 1

In this paper we show that the strong synchronization on average is equivalent to the existence of an invariant graph and a unique $\varphi$-invariant measure (see the definition below), see Theorem 1 and Theorem 3. In general, the uniqueness of a $\varphi$-invariant measure alone does not imply any type of synchronization of orbits (see Example 1 in Section 2.2 ). Thus, it is quite surprising that the existence of an invariant map "forces" the synchronization.

The strong synchronization (on average) and the existence of a measurable invariant map give rise to a globally attracting graph (on average). We show that this attractor is measurable with respect to the "past" of the random product. As a consequence we can produce a large class of random products satisfying a phenomenon called the vanishing attractor, see [1, 2] and Corollary 1 , We also use Theorem 1 to state a version of Letac's principle [17] for non i.i.d. random products on compact metric spaces, see Theorem 4 .

This paper is organized as follows. In Section 2 we state the main definitions and the precise statements of our results. In Section 3 we prove Theorem 1 and Corollary 1. In Section 4 we prove Theorems 2 and 3. Theorem 2 is used in the proof of Theorem 3 and it can be seen as a weak version of Breiman's ergodic theorem for random products. Finally, Section 5 is devoted to the proof of Theorem 4.

\footnotetext{
${ }^{1}$ Usually this fact is stated using the coordinate map $\Phi^{+}$as defined in (2.4). Note that $\Phi^{+}$ is not an invariant map, but it satisfies an invariance equation, that is, $f_{\omega}\left(\Phi^{+}(\theta(\omega))\right)=\Phi^{+}(\omega)$ which is the analogous of (1.2) for random products over non-invertible transformations.
} 


\section{MAIN RESULTS}

2.1. Existence of invariant graphs in the invertible case. In the following we take an alternative point of view of random products. A random product $\varphi$ of maps $f_{\theta^{i}(\omega)}$ on a compact metric space $X$ over a measure-preserving dynamical system $(\Omega, \mathscr{F}, \mathbb{P}, \theta)$ induces a skew product $F_{\varphi}: \Omega \times X \rightarrow \Omega \times X$ defined by

$$
F_{\varphi}(\omega, x) \stackrel{\text { def }}{=}\left(\theta(\omega), f_{\omega}(x)\right) .
$$

The map $\theta$ is called the base map and the maps $f_{\omega}$ are called the fiber maps of the skew product $F_{\varphi}$. Note that the second coordinate of $F_{\varphi}^{n}(\omega, x)$ is given by $\varphi(n, \omega, x)$.

We show that the property of strong synchronization on average implies the existence of a unique (a.e.) invariant map. Our approach is to study the space of $\varphi$-invariant measures. A probability measure $\mu$ on $\Omega \times X$ is called $\varphi$-invariant if it is $F_{\varphi}$-invariant and satisfies $\left(\Pi_{1}\right)_{*} \mu=\mathbb{P}$, where $\Pi_{1}$ is the projection on the first factor, that is, $\Pi_{1}(\omega, x)=\omega$. The space of $\varphi$-invariant measures is denoted by $I_{\mathbb{P}}(\varphi)$. We denote by $\mathcal{M}_{\mathbb{P}}(\Omega \times X)$ the space of probability measures of $\Omega \times X$ such that $\left(\Pi_{1}\right)_{*} \mu=\mathbb{P}$. We observe that $I_{\mathbb{P}}(\varphi) \neq \emptyset$ when $\Omega$ is a complete metric space, $X$ is a compact metric space and $\varphi$ is a continuous random product, that is, each map $f_{\omega}$ is continuous, see [5, Theorem 1.5.10].

In general, for random products that are synchronizing on average we cannot perform an explicit construction of the invariant map. However, we can obtain additional information of such maps. In order to precisely state what we mean by that, we need a few more definitions. Given a random variable $X$ denote by $\sigma(X)$ the $\sigma$-algebra generated by $X$. Given a random product $\varphi$ over an invertible measure-preserving dynamical system $(\Omega, \mathscr{F}, \mathbb{P}, \theta)$ the past of $\varphi$ is defined as the sub $\sigma$-algebra

$$
\mathscr{F}^{-} \stackrel{\text { def }}{=} \sigma\left(\omega \mapsto \varphi\left(n, \theta^{-n}(\omega), x\right): n \geq 1, x \in X\right),
$$

see 8 ] for details. In other words, $\mathscr{F}^{-}$is the smallest $\sigma$-algebra that makes all maps in the above family measurable.

Theorem 1. Let $\varphi$ be a random product of maps $f_{\theta^{i}(\omega)}$ on a compact metric space $X$ over an invertible measure-preserving dynamical system $(\Omega, \mathscr{F}, \mathbb{P}, \theta)$ and suppose that $I_{\mathbb{P}}(\varphi) \neq \emptyset$. If $\varphi$ is strongly synchronizing on average, then $I_{\mathbb{P}}(\varphi)$ is a singleton and there exist a unique (a.e.) invariant map $\Phi: \Omega \rightarrow X$. Moreover, the map $\Phi$ is $\mathscr{F}^{-}$-measurable and its graph is an attractor for $F_{\varphi}$ in the sense that for $\mathbb{P}$-almost every $\omega$

$$
\lim _{n \rightarrow \infty} \frac{1}{n} \sum_{i=0}^{n-1} d\left(f_{\omega}^{i}(x), \Phi\left(\theta^{i}(\omega)\right)\right)=0,
$$

for every $x \in X$.

Among the few general results about existence of invariant maps is one in [21], which we briefly describe now. Assuming that $\theta$ is a homeomorphism defined on a compact metric space $\Omega, X$ is a complete metric space and considering a random product of Lipschitz maps on $X$ over $\theta$ with maximal Lyapunov exponent (the exponential growth rate of the Lipschitz constant) bounded by a negative constant, it is proved in [21] the existence of a unique (a.e.) invariant graph. Note that the assumption on the Lyapunov exponent immediately implies exponential synchronization of orbits, that is, there is a measurable map $c: \Omega \rightarrow \mathbb{R}^{+}$and a constant 
$\rho<1$ such that for $\mathbb{P}$-almost every $\omega$ it holds

$$
d\left(f_{\omega}^{n}(x), f_{\omega}^{n}(y)\right) \leq c(\omega) \rho^{n} d(x, y)
$$

for every $x, y \in X$ and every $n \geq 0$.

In Theorem 11we only assume the existence of a $\varphi$-invariant measure and convergence of orbits on average (we do not require exponential convergence). Moreover, the maps $f_{\omega}$ are only required to be measurable and the space $\Omega$ is any measurable space.

To finish this discussion, note that 3 considered the case of a skew product with a minimal homeomorphism on a compact metric space $\Omega$ in the base and with differentiable fiber maps in $\mathbb{R}^{n}$. Considering a random compact invariant set $K \subset \Omega \times \mathbb{R}^{n}$ and assuming only that any invariant measure supported on $K$ has a negative top Lyapunov exponent it is proved that the set $K$ is the finite union of (possibly multi-valued) continuous invariant graphs. A somewhat more general setting of random minimal base maps is studied in [15].

2.1.1. The Vanishing attractor. In this section we consider i.i.d. random products of maps. Namely, let $\left(\Sigma_{0}, \mathscr{E}, \nu\right)$ be a probability space and let $\mathbb{T}$ stand for either $\mathbb{Z}=\{0, \pm 1, \pm 2, \ldots\}$ or $\mathbb{N}=\{0,1,2, \ldots\}$. Consider the space of sequences $\Sigma_{0}^{\mathbb{T}}$ endowed with the product $\sigma$-algebra $\mathscr{E}^{\mathbb{T}}$ and the product measure $\nu^{\mathbb{T}}$. Denote by $\sigma$ the shift map on the space $\Sigma_{0}^{\mathbb{T}}$, that is, $\sigma$ is defined by $\sigma\left(\left(\omega_{i}\right)_{i \in \mathbb{T}}\right)=$ $\left(\omega_{i+1}\right)_{i \in \mathbb{T}}$. The shift map is a measure-preserving transformation of the probability space $\left(\Sigma_{0}^{\mathbb{T}}, \mathscr{E}^{\mathbb{T}}, \nu^{\mathbb{T}}\right)$. The measure-preserving dynamical systems $(\Sigma, \mathscr{F}, \mathbb{P}, \sigma) \stackrel{\text { def }}{=}$ $\left(\Sigma_{0}^{\mathbb{Z}}, \mathscr{E}^{\mathbb{Z}}, \nu^{\mathbb{Z}}, \sigma\right)$ and $\left(\Sigma^{+}, \mathscr{F}^{+}, \mathbb{P}^{+}, \sigma\right) \stackrel{\text { def }}{=}\left(\Sigma_{0}^{\mathbb{N}}, \mathscr{E}^{\mathbb{N}}, \nu^{\mathbb{N}}, \sigma\right)$ are called two-sided Bernoulli shift and one-sided Bernoulli shift, respectively.

Let $X$ be a compact metric space and consider a measurable map $f: \Sigma_{0}^{\mathbb{T}} \times X \rightarrow X$ such that the map $f_{\omega}$ (recall that $f_{\omega}(x) \stackrel{\text { def }}{=} f(\omega, x)$ ) depends only on the zeroth coordinate of $\omega \in \Sigma_{0}^{\mathbb{T}}$. Then the map $\varphi: \mathbb{N} \times \Sigma_{0}^{\mathbb{T}} \times X \rightarrow X$ defined by

$$
\varphi(n, \omega, x) \stackrel{\text { def }}{=} f_{\sigma^{n-1}(\omega)} \circ \cdots \circ f_{\omega}(x)
$$

is called an independent and identically distributed (i.i.d.) random product of maps (over the one-sided or the two-sided Bernoulli shift).

In 2 the authors observe an interesting phenomenon that they call the mystery of the vanishing attractor. They present an example of an i.i.d. random product having a measurable attracting graph which vanishes when the past is forgotten, in the following sense: let $f: \Sigma \times X \rightarrow X$ be a measurable map and consider the i.i.d. random product of maps $\varphi$ over the two-sided Bernoulli shift as defined in (2.2). Let $\Pi^{+}: \Sigma \rightarrow \Sigma^{+}$be the natural projection defined by

$$
\Pi^{+}\left(\left(\omega_{i}\right)_{i \in \mathbb{Z}}\right) \stackrel{\text { def }}{=}\left(\omega_{i}\right)_{i \in \mathbb{N}}
$$

Associated with $f$ there is a unique map $f^{+}: \Sigma^{+} \times X \rightarrow X$ such that $f(\omega, x)=$ $f^{+}\left(\Pi^{+}(\omega), x\right)$ for every $(\omega, x) \in \Sigma \times X$. Hence, we can define a new i.i.d. random product of maps $\varphi^{+}$over the one-sided Bernoulli shift.

The passage from $\varphi$ to $\varphi^{+}$is understood as forgetting the past of $\varphi$, and the phenomenon of the vanishing attractor described in [1, 2] occurs when $F_{\varphi}$ has an attracting graph and $F_{\varphi^{+}}$does not. This fact may, at first, seem to be counterintuitive since attractors are limit sets of forward iterations and any orbit of a point depends only on its initial point. 
However, below we present a large class of random products having an attractor that vanishes when its past is forgotten. We show that the synchronization of orbits is a mechanism to produce such a phenomenon, because it implies that the invariant graph is measurable with respect to the past of the random product. In this way the vanishing of the attractor will be a natural consequence of the fact that in the i.i.d. case, past and future are independent.

Following 2] we consider a notion of attractor that a priori does not require invariance. Given an i.i.d. random product $\varphi$ of maps $f_{\sigma^{i}(\omega)}$ over a Bernoulli shift (two-sided or one-sided) and a measurable map $\Psi: \Sigma_{0}^{\mathbb{T}} \rightarrow X$ (not necessarily an invariant map) the basin of attraction on average of the set graph $\Psi$ is defined by

$$
\mathrm{B}_{\Psi} \stackrel{\text { def }}{=}\left\{(\omega, x) \in \Omega \times X: \lim _{n \rightarrow \infty} \frac{1}{n} \sum_{i=0}^{n-1} d\left(f_{\omega}^{i}(x), \Psi\left(\sigma^{i}(\omega)\right)\right)=0\right\} .
$$

For every $\omega$, the $\omega$-section of $\mathrm{B}_{\Psi}$ is defined by $\left(\mathrm{B}_{\Psi}\right)_{\omega} \stackrel{\text { def }}{=}\left\{x \in X:(\omega, x) \in \mathrm{B}_{\Psi}\right\}$. The set graph $\Psi$ is called an attractor on average for $F_{\varphi}$ if $\left(\mathrm{B}_{\Psi}\right)_{\omega}$ is a neighbourhood of $\Psi(\omega)$ for $\mathbb{P}$-almost every $\omega$. If $\left(B_{\Psi}\right)_{\omega}=X$ for $\mathbb{P}$-almost every $\omega$ then we say that graph $\Psi$ is a global attractor on average.

Corollary 1 (The vanishing attractor). Consider an i.i.d. random product $\varphi$ of maps $f_{\sigma^{i}(\omega)}$ on a compact metric space $X$ over the two-sided shift $(\Sigma, \mathscr{F}, \mathbb{P}, \sigma)$ and suppose that $\varphi$ is strongly synchronizing on average and the maps $f_{\omega}$ have no common fixed point $\left(\mathbb{P}\right.$-a.e.). Let $\varphi^{+}$be the associated i.i.d. random product over the one-sided Bernoulli shift $\left(\Sigma^{+}, \mathscr{F}^{+}, \mathbb{P}^{+}, \sigma\right)$. Then there is no measurable map $\Phi^{+}: \Sigma^{+} \rightarrow X$ whose graph is an attractor on average for $F_{\varphi^{+}}$.

Remark 2.1. In particular, if $\varphi$ is strongly synchronizing, that is, if for $\mathbb{P}$-almost every $\omega$ it holds

$$
\lim _{n \rightarrow \infty} d\left(f_{\omega}^{i}(x), f_{\omega}^{i}(y)\right)=0
$$

for every $x, y \in X$, then the conclusion of the corollary holds. The class of i.i.d. random products which are strongly synchronizing certainly includes i.i.d. random products of uniform contracting maps. However, there is a huge class of random product of maps without obvious contraction-like properties which are also strongly synchronizing, see [18, Corollary 2.11] and [9, Theorem 3] .

2.2. Sufficient conditions for strong synchronization. In this section we state that random products $\varphi$ on compact metric spaces having an invariant map and a unique $\varphi$-invariant measure is strongly synchronizing on average. To do so, we shall assume that the induced skew product is continuous.

We start by stablishing a version of the Breiman ergodic theorem [7] for random products having a unique $\varphi$-invariant measure. Throughout, if $\Omega$ is a compact metric space then the $\sigma$-algebra considered in any measure-preserving dynamical system $(\Omega, \mathscr{F}, \mathbb{P}, \theta)$ is the Borel $\sigma$-algebra of $\Omega$. The convergence of a sequence of Borel probability measures is always considered in the weak*-topology.

Theorem 2 (Strong Ergodic theorem). Let $\Omega$ be a compact metric space and consider a random product of maps $\varphi$ on a compact metric space $X$ over an invertible and ergodic measure-preserving dynamical system $(\Omega, \mathscr{F}, \mathbb{P}, \theta)$. Suppose that the 
skew product $F_{\varphi}$ induced by $\varphi$ is continuous and there is a unique $\varphi$-invariant measure $\mu$. Then for $\mathbb{P}$-almost every $\omega$ and every $x \in X$ we have

$$
\lim _{n \rightarrow \infty} \frac{1}{n} \sum_{i=0}^{n-1} \delta_{F_{\varphi}^{i}(\omega, x)}=\mu .
$$

Remark 2.2. Let $\varphi$ be an i.i.d. random product of maps $f_{\sigma^{i}(\omega)}$ on a compact metric space $X$ over the two-sided shift $(\Sigma, \mathscr{F}, \mathbb{P}, \sigma)$, as defined in Section 2.1.1. The family of sequences of random variables $\left(X_{n}^{x}\right)_{x \in X}$ defined by $X_{n}^{x}(\omega)=f_{\omega}^{n}(x)$ is a family of Markov chains with common transition probability, for more details see [5, Theorem 2.1.4]. If there is a unique $\varphi$-invariant measure $\mu$ then this family of Markov chains has a unique stationary measure given by $m=\Pi_{2 *} \mu$, where $\Pi_{2}$ is the natural projection on the second factor, that is, $\Pi_{2}(\omega, x)=x$ (for details see [5, Theorem 2.1.8]). Therefore, for every $x$ we can apply the Breiman ergodic theorem [7] to obtain a set $\Sigma^{x}$ with $\mathbb{P}$-full measure such that

$$
\lim _{n \rightarrow \infty} \sum_{i=0}^{n-1} \phi\left(f_{\omega}^{i}(x)\right)=\int \phi d m
$$

for every $\omega \in \Sigma^{x}$ and every continuous function $\phi: \Sigma \rightarrow \mathbb{R}$.

Let us observe that if we apply Theorem 2 to the setting above then we get a slightly stronger statement than the one obtained applying the Breiman ergodic theorem. Indeed, by Theorem 2 there is a set $\Sigma^{\prime}$ of $\mathbb{P}$-full measure such that for every $\omega \in \Sigma^{\prime}$ it holds

$\lim _{n \rightarrow \infty} \frac{1}{n} \sum_{i=0}^{n-1} \phi\left(f_{\omega}^{n}(x)\right)=\lim _{n \rightarrow \infty} \int \phi \circ \Pi_{2} d\left(\frac{1}{n} \sum_{i=0}^{n-1} \delta_{F^{i}(\omega, x)}\right)=\int \phi \circ \Pi_{2} d \mu=\int \phi d m$

for every $x \in X$ and every continuous function $\phi: X \rightarrow \mathbb{R}$.

We now stablish the main result of this section

Theorem 3. Let $\Omega$ be a compact metric space and let $\varphi$ be a random product of maps on a compact metric space $X$ over an invertible and ergodic measurepreserving dynamical system $(\Omega, \mathscr{F}, \mathbb{P}, \theta)$. Suppose that the skew product $F_{\varphi}$ induced by $\varphi$ is continuous and there are an invariant graph and a unique $\varphi$-invariant measure. Then $\varphi$ is strongly synchronizing on average.

The following example shows that the uniqueness of the $\varphi$-invariant measure alone does not imply synchronization on average.

Example 1. Let $\Omega=\{1,2\}$ endowed with the discrete $\sigma$-algebra $\mathscr{D}$ and the probability measure $\mathbb{P}=\frac{1}{2} \delta_{1}+\frac{1}{2} \delta_{2}$. Let $\theta: \Omega \rightarrow \Omega$ be the measure-preserving transformation of $(\Omega, \mathscr{D}, \mathbb{P})$ defined by $\theta(1)=2$ and $\theta(2)=1$.

Let $X=\{1,2\}$ endowed with the discrete topology and let $f: \Omega \times X \rightarrow X$ be defined by $f(1,1)=2, f(2,2)=2, f(1,2)=1$ and $f(2,1)=1$. Then, the random product $\varphi$ on $X$ over $(\Omega, \mathscr{D}, \mathbb{P}, \theta)$, whose generator is $f$, has a unique $\varphi$-invariant measure and it is not synchronizing on average.

2.3. Letac's principle for a certain class of random products. In this section we stablish a version of Letac's principle for non i.i.d. random products on a compact metric space. To recall the Letac's principle [17, let $\varphi$ be an i.i.d. continuous random product of maps $f_{\sigma^{i}(\omega)}$ on a metric space $X$ over the one-sided 
Bernoulli shift $\left(\Sigma^{+}, \mathscr{F}^{+}, \mathbb{P}^{+}, \sigma\right)$ (recall definition in Section 2.1.1). Suppose that for $\mathbb{P}^{+}$-almost every $\omega$ the limit of reversed order iterates

$$
\Phi^{+}(\omega) \stackrel{\text { def }}{=} \lim _{n \rightarrow \infty} f_{\omega} \circ \cdots \circ f_{\sigma^{n}(\omega)}(p)
$$

exists and does not depend on $p \in X$. With this assumption it is proved in [17] that the family of Markov chains $X_{n}^{x}(\omega)=f_{\omega}^{n}(\omega), x \in X$ (with common probability transition) has a unique stationary measure.

The next result can be seen as a version of Letac's principle for non i.i.d. random products. In fact, we assume a similar convergence of the reversed order iterates as in (2.4), but in a uniform way, and we obtain synchronization on average in a uniform way, too. In particular, Theorem 1 gives us the uniqueness of the invariant measure.

Theorem 4. Let $\varphi$ be a random product of maps $f_{\theta^{i}(\omega)}$ on a compact metric space $X$ over an invertible measure-preserving dynamical system $(\Omega, \mathscr{F}, \mathbb{P}, \theta)$. Then the following facts are equivalent

(1) $\lim _{n \rightarrow \infty} \operatorname{diam}\left(f_{\theta^{-1}(\omega)} \circ \cdots \circ f_{\theta^{-n}(\omega)}(X)\right)=0 \quad$ for $\mathbb{P}$-almost every $\omega$.

(2) $\lim _{n \rightarrow \infty} \frac{1}{n} \sum_{i=1}^{n} \operatorname{diam}\left(f_{\omega}^{i}(X)\right)=0 \quad$ for $\mathbb{P}$-almost every $\omega$.

Theorem 4 can be applied, for instance, to obtain the synchronization on average of a certain class of Markovian random products. In [9, Theorem 4.1] is presented a large a class of Markovian random products for which item (1) of the Theorem 4 is satisfied.

Remark 2.3. We observe that, in general, it is not possible to prove that (1) implies diam $\left(f_{\omega}^{n}(X)\right) \rightarrow 0$, see [19, Section 6].

\section{EXISTENCE OF MEASURABLE INVARIANT GRAPHS}

In this section we prove Theorem 1 and Corollary 1 . Let $(X, d)$ be a compact metric space. We first introduce a well-known tool to detect Dirac measures on $X$.

Definition 3.1. For any Borel probability measure $m$ on $X$ let

$$
D(m) \stackrel{\text { def }}{=} \iint d(x, y) d m(x) d m(y) .
$$

Lemma 3.2. If $D(m)=0$ then there is $p \in X$ such that $m=\delta_{p}$.

Proof. If $D(m)=0$, then by definition we have

$$
d(x, y)=0 \quad \text { for } \quad m \times m \text {-almost every } \quad(x, y) \in X \times X,
$$

where $m \times m$ is the product measure on $X \times X$. This implies that the support of the measure $m \times m$ is a subset of the $\operatorname{set} \operatorname{Diag}(X)=\{(x, x): x \in X\}$. Suppose that $\operatorname{supp} m$ is not a singleton. Then there are $a$ and $b$ in supp $m$ with $a \neq b$. Let $V_{1}$ and $V_{2}$ be open disjoints neighbourhoods of $a$ and $b$, respectively. Since $a$ and $b$ belong to the support of $m$, we get $m \times m\left(V_{1} \times V_{2}\right)>0$. Note also that, by the disjointness, we have $\left(V_{1} \times V_{2}\right) \cap \operatorname{Diag}(X)=\emptyset$, which contradicts the fact that the support of $m \times m$ is a subset of $\operatorname{Diag}(X)$. The proof of the lemma is now complete. 
3.1. Proof of Theorem 1. By hypothesis $I_{\mathbb{P}}(\varphi) \neq \emptyset$. We start by proving that $I_{\mathbb{P}}(\varphi)$ is a singleton. Let $\mu$ be any probability measure in $I_{\mathbb{P}}(\varphi)$ and consider its disintegration $\omega \mapsto \mu_{\omega}$ with respect to $\mathbb{P}$. The definition of $D$ and the synchronization property imply that for $\mathbb{P}$-almost every $\omega$ in $\Omega$,

$$
\lim _{n \rightarrow \infty} \frac{1}{n} \sum_{j=0}^{n-1} D\left(f_{\omega *}^{j} \mu_{\omega}\right)=\lim _{n \rightarrow \infty} \iint \frac{1}{n} \sum_{j=0}^{n-1} d\left(f_{\omega}^{n}(x), f_{\omega}^{n}(y)\right) d \mu_{\omega}(x) d \mu_{\omega}(y)=0 .
$$

Hence, using the dominated convergence theorem, we have

$$
\lim _{n \rightarrow \infty} \int \frac{1}{n} \sum_{j=0}^{n-1} D\left(f_{\omega *}^{j} \mu_{\omega}\right) d \mathbb{P}=0 .
$$

Recall that a measure $\mu$ on $\mathcal{M}_{\mathbb{P}}(\Omega \times X)$ is $\varphi$-invariant if and only if $\mu_{\theta(\omega)}=f_{\omega *} \mu_{\omega}$ for $\mathbb{P}$-almost every $\omega$ in $\Omega$, see [ 5 , Theorem 1.4.5]. In particular, for every $n \geq 1$ the equality $\mu_{\theta^{n}(\omega)}=f_{\omega *}^{n} \mu_{\omega}$ holds for $\mathbb{P}$-almost every $\omega$, and as a consequence,

$$
\int \frac{1}{n} \sum_{j=0}^{n-1} D\left(f_{\omega *}^{j} \mu_{\omega}\right) d \mathbb{P}=\int \frac{1}{n} \sum_{j=0}^{n-1} D\left(\mu_{\theta^{j}(\omega)}\right) d \mathbb{P}=\frac{1}{n} \sum_{j=0}^{n-1} \int D\left(\mu_{\theta^{j}(\omega)}\right) d \mathbb{P} .
$$

Note that, by the invariance of $\theta$, we have

$$
\int D\left(\mu_{\theta^{j}(\omega)}\right) d \mathbb{P}=\int D\left(\mu_{\omega}\right) d \mathbb{P}
$$

for every $j \geq 1$. Thus it follows from (3.1) and (3.2) that $D\left(\mu_{\omega}\right)=0$ for $\mathbb{P}$-almost every $\omega$ in $\Omega$. Applying Lemma 3.2 we get that $\mu_{\omega}$ is a delta Dirac measure for $\mathbb{P}$-almost every $\omega$, that is, there is a set $\Lambda \subset \Omega$ with $\mathbb{P}$-full measure and a map $\Phi: \Lambda \rightarrow X$ such that

$$
\mu_{\omega}=\delta_{\Phi(\omega)}
$$

for every $\omega \in \Lambda$.

This shows that the disintegration of any $\varphi$-invariant measure is atomic. This fact implies that $I_{\mathbb{P}}(\varphi)$ is a singleton. Indeed, let $\mu$ and $\nu$ be two $\varphi$-invariant measures. Then there are maps $\Phi, \Psi: \Omega \rightarrow X$ such that $\omega \mapsto \delta_{\Phi(\omega)}$ and $\omega \mapsto \delta_{\Psi(\omega)}$ are the disintegration of $\mu$ and $\nu$, respectively. The probability measure $\frac{\mu+\nu}{2}$ is also a $\varphi$-invariant measure and then there exists a map $\Gamma: \Omega \rightarrow X$ such that the disintegration of $\frac{\mu+\nu}{2}$ is given by $\omega \mapsto \delta_{\Gamma(\omega)}$. On the other hand, $\omega \mapsto \frac{\delta_{\Phi(\omega)}+\delta_{\Psi(\omega)}}{2}$ is also a disintegration of $\frac{\mu+\nu}{2}$. By the uniqueness (a.e.) of the disintegration of a probability measure we have

$$
\frac{\delta_{\Phi(\omega)}+\delta_{\Psi(\omega)}}{2}=\delta_{\Gamma(\omega)}
$$

for $\mathbb{P}$-almost every $\omega$ in $\Omega$, which implies that $\Gamma(\omega)=\Phi(\omega)=\Psi(\omega)$ for $\mathbb{P}$-almost every $\omega$ in $\Omega$. Therefore, $I_{\mathbb{P}}(\varphi)$ is a singleton.

Let $\mu$ be the unique $\varphi$-invariant measure and let $\Phi: \Omega \rightarrow X$ be a map such that $\omega \mapsto \delta_{\Phi(\omega)}$ is the disintegration of $\mu$. We claim that $\Phi$ is an invariant map. We start by proving that $\Phi$ is measurable: for every Borel measurable set $R \subset X$ we have $\gamma_{R}^{-1}(\{1\})=\Phi^{-1}(R)$, where $\gamma_{R}$ is the map $\omega \mapsto \mu_{\omega}(R)$. By the definition of disintegrations we have that $\gamma_{R}$ is measurable, which implies that $\Phi$ is also measurable. 
To prove that $\Phi$ is an invariant map recall that the $\varphi$-invariance of $\mu$ implies $\mu_{\theta(\omega)}=f_{\omega *} \mu_{\omega}$ for $\mathbb{P}$-almost every $\omega$ in $\Omega$. Hence we have

$$
\delta_{\Phi(\theta(\omega))}=\mu_{\theta(\omega)}=f_{\omega *} \mu_{\omega}=f_{\omega *} \delta_{\Phi(\omega)}=\delta_{f_{\omega}(\Phi(\omega))}
$$

for $\mathbb{P}$-almost every $\omega$ in $\Omega$, which implies that $f_{\omega}(\Phi(\omega))=\Phi(\theta(\omega))$ for $\mathbb{P}$-almost every $\omega$ in $\Omega$. The uniqueness (a.e.) of the invariant map is proved observing that every invariant map determines a $\varphi$-invariant measure, as in (3.3).

We now prove that the graph of $\Phi$ is a global attractor on average. First note that the invariance of $\Phi$ implies that for every $n \geq 0$

$$
\Phi\left(\theta^{n}(\omega)\right)=f_{\omega}^{n}(\Phi(\omega)),
$$

for $\mathbb{P}$-almost every $\omega$ in $\Omega$. Thus, it follows from the synchronization property that for $\mathbb{P}$-almost every $\omega$ in $\Omega$ we have

$$
\lim _{n \rightarrow \infty} \frac{1}{n} \sum_{i=0}^{n-1} d\left(f_{\omega}^{i}(x), \Phi\left(\theta^{i}(\omega)\right)\right)=0
$$

for every $x \in X$.

Finally, we prove that $\Phi$ is $\mathscr{F}^{-}$-measurable. From (3.4) and the dominated convergence theorem we have

$$
\lim _{n \rightarrow \infty} \int \frac{1}{n} \sum_{i=0}^{n-1} d\left(f_{\omega}^{i}(x), \Phi\left(\theta^{i}(\omega)\right) d \mathbb{P}=0,\right.
$$

for every $x \in X$. Take any $x \in X$, by the invariance of $\theta$ it follows from (3.5) that

$$
\lim _{n \rightarrow \infty} \frac{1}{n} \sum_{i=0}^{n-1} \int d\left(f_{\theta^{-i}(\omega)}^{i}(x), \Phi(\omega)\right) d \mathbb{P}=0 .
$$

Since $d\left(f_{\theta^{-i}(\omega)}^{i}(x), \Phi(\omega)\right) \geq 0$ for every $i \geq 0$, we obtain from (3.6) that there exists a subsequence $\left(n_{k}\right)_{k}$ such that

$$
\lim _{k \rightarrow \infty} \int d\left(f_{\theta^{-n_{k}(\omega)}}^{n_{k}}(x), \Phi(\omega)\right) d \mathbb{P}=0 .
$$

Thus, passing to another subsequence if necessary we have

$$
\Phi(\omega)=\lim _{k \rightarrow \infty} f_{\theta^{-n_{k}(\omega)}}^{n_{k}}(x),
$$

for $\mathbb{P}$-almost every $\omega$ in $\Omega$, which implies that $\Phi$ is $\mathscr{F}^{-}$-measurable. The proof of the theorem is now complete.

Remark 3.3. Note that in the above proof we needed to prove first that $\Phi$ is measurable because to prove that it is $\mathscr{F}^{-}$-measurable we take an integral involving this map, see (3.5).

3.2. Proof of Corollary 1, By Theorem 1 there exists a $\mathscr{F}^{-}$-measurable map $\Phi: \Sigma \rightarrow X$ whose graph is a global attractor on average.

Suppose that there is a measurable map $\Phi^{+}: \Sigma^{+} \rightarrow X$ whose graph is an attractor on average. Let $\Pi^{+}: \Sigma \rightarrow \Sigma^{+}$be the natural projection defined in (2.3).

Claim 3.4. $\Phi(\omega)=\Phi^{+} \circ \Pi^{+}(\omega)$ for $\mathbb{P}$-almost every $\omega$. 
Proof. By hypothesis, the graph of $\Phi^{+}$is an attractor on average for $F_{\varphi^{+}}$. Noting that $\Pi_{*}^{+} \mathbb{P}=\mathbb{P}^{+}$, we get that the graph of $\Phi^{+} \circ \Pi^{+}$is an attractor on average for $F_{\varphi}$. Observe that for every $x$ and $\omega$ we have

$$
d\left(\Phi\left(\theta^{i}(\omega)\right), \Phi^{+} \circ \Pi^{+}\left(\theta^{i}(\omega)\right)\right) \leq d\left(f_{\omega}^{i}(x), \Phi\left(\theta^{i}(\omega)\right)\right)+d\left(f_{\omega}^{i}(x), \Phi^{+} \circ \Pi^{+}\left(\theta^{i}(\omega)\right)\right),
$$

for every $i \geq 0$. Since the graph of $\Phi$ is a global attractor on average, for $\mathbb{P}$-almost every $\omega$ we can take a point $x(\omega) \in X$ such that

$$
x(\omega) \in \rho(\Phi)_{\omega} \cap \rho\left(\Phi^{+} \circ \Pi^{+}\right)_{\omega} .
$$

Applying (3.7) to the point $x(\omega)$ we obtain that for $\mathbb{P}$-almost every $\omega$

$$
\lim _{n \rightarrow \infty} \frac{1}{n} \sum_{i=0}^{n-1} d\left(\Phi\left(\theta^{i}(\omega)\right), \Phi^{+} \circ \Pi^{+}\left(\theta^{i}(\omega)\right)=0 .\right.
$$

Using the dominated convergence theorem and the invariance of $\theta$ we conclude that

$$
\begin{aligned}
0 & =\lim _{n \rightarrow \infty} \int \frac{1}{n} \sum_{i=0}^{n-1} d\left(\Phi\left(\theta^{i}(\omega)\right), \Phi^{+} \circ \Pi^{+}\left(\theta^{i}(\omega)\right) d \mathbb{P}(\omega)\right. \\
& =\lim _{n \rightarrow \infty} \frac{1}{n} \sum_{i=0}^{n-1} \int d\left(\Phi(\omega), \Phi^{+} \circ \Pi^{+}(\omega)\right) d \mathbb{P}(\omega)=\int d\left(\Phi(\omega), \Phi^{+} \circ \Pi^{+}(\omega)\right) d \mathbb{P}(\omega) .
\end{aligned}
$$

Since $d\left(\Phi(\omega), \Phi^{+} \circ \Pi^{+}(\omega)\right) \geq 0$ for every $\omega$, we obtain from (3.8) that $\Phi(\omega)=$ $\Phi^{+} \circ \Pi^{+}(\omega)$ for $\mathbb{P}$-almost every $\omega$, ending the proof of the claim.

In order to conclude the proof of Corollary 1 we consider the future of the random product $\varphi$ defined by

$$
\mathscr{F}^{+} \stackrel{\text { def }}{=} \sigma\left(\omega \mapsto \varphi\left(n, \theta^{n}(\omega), x\right): n \geq 0, x \in X\right) .
$$

Note that $\Phi^{+} \circ \Pi^{+}$is $\mathscr{F}^{+}$-measurable. On the other hand, $\Phi$ is $\mathscr{F}^{-}$-measurable. Since $\mathscr{F}^{+}$and $\mathscr{F}^{-}$are independent $\sigma$-algebras we obtain that $\Phi$ is constant $\mathbb{P}$ almost everywhere. Indeed, given a measurable set $A \in X$, by independence we have

$$
\begin{aligned}
\mathbb{P}\left(\Phi^{-1}(A)\right) & =\mathbb{P}\left(\Phi^{-1}(A) \cap\left(\Phi^{+} \circ \Pi^{+}\right)^{-1}(A)\right) \\
& =\mathbb{P}\left(\Phi^{-1}(A)\right) \mathbb{P}\left(\left(\Phi^{+} \circ \Pi^{+}\right)^{-1}(A)\right)=\mathbb{P}\left(\Phi^{-1}(A)\right)^{2},
\end{aligned}
$$

which implies that $\mathbb{P}\left(\Phi^{-1}(A)\right)=0$ or $\mathbb{P}\left(\Phi^{-1}(A)\right)=1$. Thus, the $\sigma$-algebra generated by $\Phi$ coincides $(\bmod 0)$ with the trivial $\sigma$-algebra $\{\emptyset, \Omega\}$, which implies that $\Phi$ is $\mathbb{P}$-a.e. constant, say, $\Phi(\omega)=p$ for $\mathbb{P}$-almost every $\omega$ for some $p \in X$. Since $\Phi$ is an invariant map, we obtain $f_{\omega}(p)=p$ for $\mathbb{P}$-almost every $\omega$, which contradicts the hypothesis. Therefore, there is no measurable map $\Phi^{+}$whose graph is an attractor on average, ending the proof of the corollary.

\section{Sufficient CONDition FOR SynChronizAtion on AVERAGE}

In this section we prove Theorems 2 and 3 . 
4.1. Proof of Theorem 2. Let $\Omega_{\theta}$ be a subset of $\Omega$ such that for every $\omega \in \Omega_{\theta}$ we have

$$
\lim _{n \rightarrow \infty} \frac{1}{n} \sum_{i=0}^{n-1} \delta_{\theta^{i}(\omega)}=\mathbb{P} .
$$

Since $\theta$ is ergodic we deduce that $\mathbb{P}\left(\Omega_{\theta}\right)=1$. Let $F$ be the skew product associated with $\varphi$, recall (2.1). We claim that for $(\omega, x) \in \Omega_{\theta} \times X$ it holds

$$
\lim _{n \rightarrow \infty} \frac{1}{n} \sum_{i=0}^{n-1} \delta_{F^{i}(\omega, x)}=\mu,
$$

where $\mu$ is the unique $\varphi$-invariant measure. To see why this is so, let $\Pi_{1}: \Omega \times X \rightarrow \Omega$ be the natural projection on the first factor. Then for every $(\omega, x)$ it holds

$$
\Pi_{1 *}\left(\frac{1}{n} \sum_{i=0}^{n-1} \delta_{F^{i}(\omega, x)}\right)=\frac{1}{n} \sum_{i=0}^{n-1} \delta_{\theta^{i}(\omega)} .
$$

Hence, it follows from (4.1) and the continuity of $\Pi_{1 *}$ (in the weak*- topology) that for every $(\omega, x) \in \Omega_{\theta} \times X$ any accumulation point $\mu^{\prime}$ of $\frac{1}{n} \sum_{i=0}^{n-1} \delta_{F^{i}(\omega, x)}$ satisfies $\Pi_{1 *} \mu^{\prime}=\mathbb{P}$. Since any accumulation point of $\frac{1}{n} \sum_{i=0}^{n-1} \delta_{F^{i}(\omega, x)}$ is a $F$-invariant measure then it follows from the uniqueness of $\varphi$-invariant measures that

$$
\lim _{n \rightarrow \infty} \frac{1}{n} \sum_{i=0}^{n-1} \delta_{F^{i}(\omega, x)}=\mu,
$$

for every $(\omega, x) \in \Omega_{\theta} \times X$.

4.2. Proof of Theorem 3. Suppose that there exists a unique $\varphi$-invariant measure $\mu$ and assume the existence of a measurable invariant map $\Phi: \Omega \rightarrow X$. Let $g: \Omega \times$ $X \rightarrow \mathbb{R}$ be the map defined by

$$
g(\omega, x) \stackrel{\text { def }}{=} d(x, \Phi(\omega))
$$

Since $\omega \mapsto d(x, \Phi(\omega))$ is measurable for every $x$ and $x \mapsto d(x, \Phi(\omega))$ is continuous for every $\omega$, Carathéodorys theorem implies that the function $g$ is $\mathscr{F} \otimes \mathscr{B}$-measurable, see [8]. The following preliminary result is the key step to the proof of Theorem 3

Lemma 4.1. For $\mathbb{P}$-almost every $\omega$ in $\Omega$ we have

$$
\lim _{n \rightarrow \infty} \int g d \mu_{n}^{\omega, x}=0
$$

for every $x \in X$, where $\mu_{n}^{\omega, x}=\frac{1}{n} \sum_{i=0}^{n-1} \delta_{F^{i}(\omega, x)}$.

Proof. Since $\Omega$ is a compact metric space, given $k>0$ it follows from Lusin's theorem that there exists a compact set $F_{k} \subset \Omega$ such that $\Phi_{\mid F_{k}}$ is a continuous map and $\mathbb{P}\left(F_{k}^{c}\right)<\frac{1}{k}$. Note that $g$ is continuous on $F_{k} \times X$.

Claim 4.2. $g \mathbb{1}_{F_{k} \times X}$ is upper semi-continuous.

Proof. Given $\alpha>0$ we need to see that the set $A_{\alpha}=\{(\omega, x) \in \Omega \times X: g(\omega, x)<\alpha\}$ is an open set. If $A_{\alpha}$ is not empty, given a point $(\xi, y) \in A_{\alpha}$, since $g_{\mid F_{k} \times X}$ is continuous there is an open neighbourhood $V$ of $(\xi, y)$ such that

$$
g \mathbb{1}_{F_{k} \times X}(\omega, x)=g(\omega, x)<\alpha \quad \text { for every } \quad(\omega, x) \in V \cap F_{k} \times X .
$$


On the other hand, if $(\omega, x) \notin F_{k} \times X$ then $g \mathbb{1}_{F_{k} \times X}(\omega, x)=0$, proving the claim.

By Theorem 2, there exists a set $\Lambda \subset \Omega$ with $\mathbb{P}$-full measure such that $\lim _{n \rightarrow \infty} \mu_{n}^{\omega, x}=$ $\mu$ for every $(\omega, x) \in \Lambda \times X$. Then, it follows from Alexandrov's theorem and Claim 4.2 that

$$
\limsup _{n \rightarrow \infty} \int g \mathbb{1}_{F_{k} \times X} d \mu_{n}^{\omega, x} \leq \int g \mathbb{1}_{F_{k} \times X} d \mu \leq \int g d \mu
$$

for every $(\omega, x) \in \Lambda \times X$.

Now let us compute $\int g d \mu$. The uniqueness of $\mu$ and the invariance of $\Phi$ implies that the disintegration of $\mu$ with respect to $\mathbb{P}$ is given by $\omega \mapsto \delta_{\Phi(\omega)}$. Hence,

$$
\begin{aligned}
\int g(\omega, x) d \mu(\omega, x) & =\int_{\Omega}\left(\int_{X} g(\omega, x) d \delta_{\Phi(\omega)}(x)\right) d \mathbb{P}(\omega) \\
& =\int_{\Omega} g(\omega, \Phi(\omega)) d \mathbb{P}(\omega)=0 .
\end{aligned}
$$

Thus, by (4.2) we deduce that $\lim \sup _{n \rightarrow \infty} \int g \mathbb{1}_{F_{k} \times X} d \mu_{n}^{\omega, x}=0$ for every $(\omega, x) \in$ $\Lambda \times X$.

Since $X$ is a compact metric space, we have $L \stackrel{\text { def }}{=} \sup _{x, y \in X} d(x, y)<\infty$. Hence for every $(\omega, x) \in \Lambda \times X$ we have

$$
\begin{aligned}
\limsup _{n \rightarrow \infty} \int g d \mu_{n}^{\omega, x} & =\limsup _{n \rightarrow \infty}\left(\int_{F_{k} \times X} g d \mu_{n}^{\omega, x}+\int_{F_{k}^{c} \times X} g d \mu_{n}^{\omega, x}\right) \\
& \leq \limsup _{n \rightarrow \infty} \int_{F_{k} \times X} g d \mu_{n}^{\omega, x}+\limsup _{n \rightarrow \infty} \int_{F_{k}^{c} \times X} g d \mu_{n}^{\omega, x} \\
& \leq L \limsup _{n \rightarrow \infty} \mu_{n}^{\omega, x}\left(F_{k}^{c} \times X\right) .
\end{aligned}
$$

Claim 4.3. There exists a set $\Lambda^{\prime} \subset \Omega$ with $\mathbb{P}$-full measure such that

$$
\lim _{n \rightarrow \infty} \mu_{n}^{\omega, x}\left(F_{k}^{c} \times X\right)=\mathbb{P}\left(F_{k}^{c}\right)
$$

for every $\omega \in \Lambda^{\prime}$, every $x \in X$ and every $k \geq 1$.

Proof. It follows from the definition of $\mu_{n}^{\omega, x}$ that for every $(\omega, x) \in \Omega \times X$ and every $k \geq 1$ we have

$$
\mu_{n}^{\omega, x}\left(F_{k}^{c} \times X\right)=\frac{1}{n} \sum_{i=0}^{n-1} \delta_{\theta^{i}(\omega)}\left(F_{k}^{c}\right) .
$$

By Birkhoff's ergodic theorem there is a set $\Lambda_{k}$ with $\mathbb{P}\left(\Lambda_{k}\right)=1$ and such that for every $\omega \in \Lambda_{k}$ it holds

$$
\lim _{n \rightarrow \infty} \frac{1}{n} \sum_{i=0}^{n-1} \delta_{\theta^{i}(\omega)}\left(F_{k}^{c}\right)=\mathbb{P}\left(F_{k}^{c}\right) .
$$

Thus, defining $\Lambda^{\prime} \stackrel{\text { def }}{=} \cap_{k \geq 1} \Lambda_{k}$ we have

$$
\lim _{n \rightarrow \infty} \mu_{n}^{\omega, x}\left(F_{k}^{c} \times X\right)=\mathbb{P}\left(F_{k}^{c}\right)
$$

for every $(\omega, x) \in \Lambda^{\prime} \times X$ and every $k \geq 1$, ending the proof of the claim. 
Now, setting $\Omega^{\prime} \stackrel{\text { def }}{=} \Lambda^{\prime} \cap \Lambda$, it follows from (4.3) and Claim 4.3 that

$$
\limsup _{n \rightarrow \infty} \int g d \mu_{n}^{\omega, x} \leq L \mathbb{P}\left(F_{k}^{c}\right) \leq L \frac{1}{k}
$$

for every $(\omega, x) \in \Omega^{\prime} \cap X$ and every $k \geq 1$. Hence, from (4.3) we have

$$
\lim _{n \rightarrow \infty} \int g d \mu_{n}^{\omega, x}=0
$$

for every $(\omega, x) \in \Omega^{\prime} \cap X$. The proof of the lemma is now complete.

We now conclude the proof of the theorem. First, note that for every $\omega \in \Omega$ and every pair $x, y \in X$ we have

$$
\frac{1}{n} \sum_{i=0}^{n-1} d\left(f_{\omega}^{i}(x), f_{\omega}^{i}(y)\right) \leq \frac{1}{n} \sum_{i=0}^{n-1} d\left(f_{\omega}^{j}(x), \Phi\left(\theta^{j}(\omega)\right)\right)+\frac{1}{n} \sum_{i=0}^{n-1} d\left(f_{\omega}^{j}(y), \Phi\left(\theta^{j}(\omega)\right)\right),
$$

and it follows from the definition of $\mu_{n}^{\omega, z}$ that

$$
\int g d \mu_{n}^{\omega, z}=\frac{1}{n} \sum_{i=0}^{n-1} d\left(f_{\omega}^{j}(z), \Phi\left(\theta^{j}(\omega)\right)\right)
$$

for every $\omega$ and every $z \in X$. Then from Lemma 4.1 we deduce that for $\mathbb{P}$-almost every $\omega$ in $\Omega$

$$
\lim _{n \rightarrow \infty} \frac{1}{n} \sum_{i=0}^{n-1} d\left(f_{\omega}^{i}(x), f_{\omega}^{i}(y)\right)=0,
$$

for every $x, y \in X$. The proof of the theorem is now complete.

\section{Proof of Theorem 4}

We first prove that $(1) \Rightarrow(2)$. For that, consider the sequence of measurable functions $g_{n}: \Omega \rightarrow[0,+\infty)$ defined by $g_{n}(\omega) \stackrel{\text { def }}{=} \operatorname{diam}\left(f_{\omega}^{n}(X)\right), n \geq 1$, and observe that,

$$
\begin{aligned}
g_{n+1}(\omega) & =\operatorname{diam}\left(f_{\theta^{n}(\omega)} \circ \cdots \circ f_{\omega}(X)\right) \\
& =\operatorname{diam}\left(f_{\theta^{n+1}(\omega)} \circ \cdots \circ f_{\theta(\omega)}\left(f_{\omega}(X)\right)\right) \\
& \leq g_{n}(\theta(\omega)),
\end{aligned}
$$

for every $\omega \in \Omega$ and every $n \geq 1$. Hence, for $m, k \in \mathbb{N}$ we have $g_{m+k}(\omega) \leq$ $g_{m}\left(\theta^{k}(\omega)\right)$. Define $u_{n}(\omega)=\sum_{j=1}^{n} g_{j}(\omega)$, and note that

$$
u_{m+n}=\sum_{j=1}^{m+n} g_{j}=u_{m}+\sum_{j=m+1}^{m+n} g_{j} \leq u_{m}+\sum_{i=1}^{n} g_{i} \circ \theta^{m}=u_{m}+u_{n} \circ \theta^{m},
$$

which implies that the sequence $\left(u_{n}\right)_{n}$ is subadditive. Thus, by Kingman's subadditive ergodic theorem the sequence $\left(u_{n} / n\right)_{n}$ converges $\mathbb{P}$-almost everywhere to a non-negative invariant function $g$ such that

$$
\int g d \mathbb{P}=\lim _{n \rightarrow \infty} \frac{1}{n} \int u_{n} d \mathbb{P} .
$$


Now consider the sequence of measurable functions $h_{n}: \Omega \rightarrow[0,+\infty)$ defined by

$$
h_{n}(\omega) \stackrel{\text { def }}{=} \operatorname{diam} f_{\theta^{-n}(\omega)}^{n}(X)=\operatorname{diam} f_{\theta^{-1}(\omega)} \circ \cdots \circ f_{\theta^{-n}(\omega)}(X)
$$

By hypothesis, we have that $\lim _{n \rightarrow \infty} h_{n}(\omega)=0$ for $\mathbb{P}$-almost every $\omega$. In particular, it follows from the dominated convergence theorem that

$$
\lim _{n \rightarrow+\infty} \frac{1}{n} \sum_{j=1}^{n} \int h_{j} d \mathbb{P}=0,
$$

for $\mathbb{P}$-almost every $\omega$. Also, noting that $g_{n} \circ \theta^{-n}=h_{n}$, and using the invariance of $\theta$ we have

$$
\int h_{i} d \mathbb{P}=\int g_{i} d \mathbb{P}
$$

for every $i \geq 1$. Therefore, from (5.1) and (5.2) we get

$$
\begin{aligned}
\int g d \mathbb{P} & =\lim _{n \rightarrow+\infty} \frac{1}{n} \int u_{n} d \mathbb{P} \\
& =\lim _{n \rightarrow+\infty} \frac{1}{n} \sum_{j=1}^{n} \int g_{j} d \mathbb{P}=\lim _{n \rightarrow+\infty} \frac{1}{n} \sum_{j=1}^{n} \int h_{j} d \mathbb{P}=0 .
\end{aligned}
$$

Since $g(\omega) \geq 0$ for $\mathbb{P}$-almost every $\omega$, we conclude that $g(\omega)=0$ for $\mathbb{P}$-almost every $\omega$, which proves that $(1) \Rightarrow(2)$.

We now prove that $(2) \Rightarrow(1)$. First note that $h_{n+1}(\omega) \leq h_{n}(\omega)$ for every $n \geq 1$ and $\omega \in \Omega$. Hence the limit

$$
h(\omega) \stackrel{\text { def }}{=} \lim _{n \rightarrow \infty} h_{n}(\omega)
$$

exists for every $\omega \in \Omega$. In particular, using the dominated convergence theorem and (5.3) we have

$$
\int h(\omega) d \mathbb{P}=\lim _{n \rightarrow \infty} \int \frac{1}{n} \sum_{i=1}^{n} h_{i} d \mathbb{P}=\lim _{n \rightarrow \infty} \int \frac{1}{n} \sum_{i=1}^{n} g_{i} d \mathbb{P}=0,
$$

where the last equality follows from the hypothesis $\lim _{n \rightarrow \infty} \frac{1}{n} \sum_{i=1}^{n} g_{i}(\omega)=0$ for $\mathbb{P}$-almost every $\omega$. Since $h(\omega) \geq 0$ for every $\omega$, we conclude that $h(\omega)=0$ for $\mathbb{P}$-almost every $\omega$, which completes the proof of $(2) \Rightarrow(1)$.

\section{REFERENCES}

[1] Alsedì, L. S., And Misiurewicz, M. Random interval homeomorphisms. Publ. Mat. 58, suppl. (2014), 15-36.

[2] Alsedì, L. S., And Misiurewicz, M. Skew product attractors and concavity. Proc. Amer. Math. Soc. 143, 2 (2015), 703-716.

[3] Anagnostopoulou, V., And JäGer, T. Nonautonomous saddle-node bifurcations: random and deterministic forcing. J. Differential Equations 253, 2 (2012), 379-399.

[4] Antonov, V. A. Modeling of processes of cyclic evolution type. Synchronization by a random signal. Vestnik Leningrad. Univ. Mat. Mekh. Astronom., vyp. 2 (1984), 67-76.

[5] Arnold, L. Random dynamical systems. Springer Monographs in Mathematics. SpringerVerlag, Berlin, 1998.

[6] Barnsley, M. F., Elton, J. H., and Hardin, D. P. Recurrent iterated function systems. Constr. Approx. 5, 1 (1989), 3-31.

[7] Breiman, L. The strong law of large numbers for a class of Markov chains. Ann. Math. Statist. 31 (1960), 801-803. 
[8] Crauel, H. Random probability measures on Polish spaces, vol. 11 of Stochastics Monographs. Taylor \& Francis, London, 2002.

[9] Díaz, L. J., And Matias, E. Stability of the markov operator and synchronization of markovian random products. arXiv:1707.06411.

[10] Furstenberg, H. Noncommuting random products. Trans. Amer. Math. Soc. 108 (1963), $377-428$.

[11] Gelfert, K., And Stenflo, O. Random iterations of homeomorphisms on the circle. Mod. Stoch. Theory Appl. 4 (2017), 253-271.

[12] Gorodetski, A., And Kleptsyn, V. Synchronization properties of random piecewise isometries. Comm. Math. Phys. 345, 3 (2016), 781-796.

[13] Hugenir (Huygens), C. H. Horologium Oscillatorium. Parisiis, France, (1673).

[14] Hutchinson, J. E. Fractals and self-similarity. Indiana Univ. Math. J. 30, 5 (1981), 713-747.

[15] JÄGER, T., AND KelLER, G. Random minimality and continuity of invariant graphs in random dynamical systems. Trans. Amer. Math. Soc. 368, 9 (2016), 6643-6662.

[16] Kleptsyn, V. A., And NAL'SkiI, M. B. Convergence of orbits in random dynamical systems on a circle. Funktsional. Anal. i Prilozhen. 38, 4 (2004), 36-54, 95-96.

[17] Letac, G. A contraction principle for certain Markov chains and its applications. In Random matrices and their applications (Brunswick, Maine, 1984), vol. 50 of Contemp. Math. Amer. Math. Soc., Providence, RI, 1986, pp. 263-273.

[18] Malicet, D. Random Walks on Homeo( $\left.S^{1}\right)$. Comm. Math. Phys. 356, 3 (2017), 1083-1116.

[19] Melo, Í. On $\mathbb{P}$-weakly hyperbolic iterated function system. Bull. Braz. Math. Soc. 48, 4 (2017), 717-732.

[20] Pikovsky, A., Rosenblum, M., and Kurths, J. Synchronization, vol. 12 of Cambridge Nonlinear Science Series. Cambridge University Press, Cambridge, 2001. A universal concept in nonlinear sciences.

[21] Stark, J. Regularity of invariant graphs for forced systems. Ergodic Theory Dynam. Systems 19, 1 (1999), 155-199.

Instituto de Matemática, Universidade Federal do Rio de Janeiro, Av. Athos da Silveira Ramos 149, Cidade Universitária - Ilha do Fundão, Rio de Janeiro 21945-909, BRAZIL

E-mail address: edgarmatias9271@gmail.com

Universidade Federal do Piauí, Departamento de Matemática, 64049-550, Ininga TERESINA - PI, BRAZIL

E-mail address: italodowell@ufpi.edu.br 